\title{
Desempenho de idosos com e sem sintomas depressivos no WCST-64
}

\author{
Cristiane Silva Esteves², Camila Rosa de Oliveira, Tatiana Quarti Irigaray, Irani Iracema de Lima Argimon \\ Pontifícia Universidade Católica do Rio Grande do Sul, Porto Alegre-RS, Brasil
}

\section{RESUMO}

É natural que idosos apresentem dificuldades nas funções executivas mesmo nos estágios iniciais de um quadro depressivo, que podem ser detectadas pelo Teste Wisconsin de Classificação de Cartas (WCST). O objetivo do presente estudo foi comparar o desempenho de idosos com e sem sintomas depressivos no WCST, versão abreviada de 64 cartas (WCST-64). A amostra foi composta por 153 idosos, divididos em dois grupos: com e sem sintomas depressivos. Os grupos foram pareados em relação à idade, escolaridade e escore no Mini Exame do Estado Mental (MEEM). Utilizou-se uma ficha de dados sociodemográficos, a Escala de Depressão Geriátrica, o MEEM e o WCST-64. Observou-se que, na medida em que os sintomas de depressão se intensificavam, aumentavam o total de erros, as respostas perseverativas e os erros perseverativos no WCST-64. Os resultados mostraram que idosos que apresentam sintomas depressivos tiveram pior desempenho no WCST-64, quando comparados com aqueles sem sintomatologia depressiva.

Palavras-chave: depressão; idoso; envelhecimento; teste Wisconsin de Classificação de Cartas; função executiva.

\section{ABSTRACT - Elderly performance with and without depressive symptoms on the WCST-64}

It is natural that the elderly would present difficulties in executive functions even in the early stages of a depressive condition, which may be detected by the Wisconsin Card Sorting Test (WCST). This study aimed to compare the performance of elderly people with and without depressive symptoms on the shortened version of the WCST, containing 64 cards (WCST-64). The sample consisted of 153 elderly subjects who were divided into two groups: with and without depressive symptoms. The groups were matched in terms of age, educational level and score on the Mini Mental State Examination (MMSE). A Socio-Demographic Data Sheet, the Geriatric Depression Scale, MMSE and the WCST-64 were used. It was observed that, to the extent that depressive symptoms intensified, total errors, perseverative responses and perseverative errors increased on the WCST-64. The results showed that elderly people who present depressive symptoms had poorer performance on the WCST-64, compared to those without depressive symptoms. Keywords: depression; elderly people; aging; Wisconsin Card Sorting Test; executive function.

\section{RESUMEN - Rendimiento de ancianos con y sin síntomas depresivos em WCST-64}

En las etapas iniciales de un cuadro de depresión es natural que los ancianos presenten dificultades en las funciones ejecutivas, que pueden ser detectadas por el Test de Wisconsin de Clasificación de Cartas (WCST). El objetivo de este estudio fue comparar el desempeño de las personas mayores con y sin síntomas de depresión en el WCST, versión abreviada de 64 cartas (WCST-64). La muestra estuvo constituída por 153 ancianos divididos en dos grupos: con y sin síntomas de depresión. Los grupos fueron comparados por edad, nivel educativo y resultado en el Mini Mental State Examination (MMSE). Se utilizó una ficha de datos sociodemográficos, la Escala de Depresión Geriátrica, MMSE y el WCST-64. A medida que los síntomas de depresión se intensificaban, aumentaba el número total de errores, respuestas perseverativos y errores perseverativos en el WCST-64. Los resultados mostraron que los ancianos que presentan síntomas de depresión tuvieron peor desempeño en el WCST-64, comparados con aquellos que no poseían esos síntomas.

Palabras clave: depresión; ancianos; envejecimiento; Test Wisconsin de Clasificación de Cartas; función ejecutiva.

Os transtornos de humor têm sido considerados problemas de saúde pública (Fernandes, Falcone, \& Sardinha, 2012; Santos \& Siqueira, 2010), sendo a depressão o mais prevalente em idosos, atingindo de $4,8 \%$ a $14,6 \%$ dos que vivem na comunidade (Irigaray \& Schneider, 2007) e, anualmente, afetando em torno de
$5 \%$ da população mundial (Garcia et al., 2006). Na velhice, muitas vezes, a depressão é subdiagnosticada, por suas características acabarem sendo confundidas com os sintomas naturais do processo de envelhecimento (Gunn et al., 2012). A causa da depressão é multifatorial e envolve variáveis psicológicas, socioculturais e biológicas

${ }^{1}$ Apoio institucional ao projeto: CAPES.

2 Endereço para correspondência: Pontifícia Universidade Católica do Rio Grande do Sul, Prédio 11, Sala 914, Avenida Ipiranga, 6681, 90619-900, Porto Alegre-RS.

E-mail: crissilvaesteves@gmail.com 
(Fernandes et al., 2012), podendo causar diversos prejuízos na área cognitiva e no desempenho social. Sendo assim, o diagnóstico de depressão pode trazer, como características, modificações nas habilidades cognitivas, que incluem alterações nas funções executivas, na memória, principalmente, de trabalho, além da diminuição da velocidade do processamento da informação (Steibel \& Almeida, 2010; Xavier, 2006).

Steibel e Almeida (2010) supõem as seguintes hipóteses que podem explicar a associação entre depressão e perdas cognitivas: (a) o estado depressivo pode se dar como uma reação emocional ao diagnóstico ou à percepção de um início de quadro demencial; (b) uma possível causa comum que ocorra no sistema nervoso central que leve tanto ao declínio das habilidades cognitivas, quanto à depressão com o envelhecimento. Além disso, ainda existe a possibilidade de que sejam doenças comórbidas, ou seja, de que não haja nenhuma relação de causa e efeito entre ambas. Ávila e Bottino (2006) também apontam que o quadro depressivo pode ocorrer em comorbidade com déficits cognitivos. Ou seja, as alterações no desempenho cognitivo de idosos podem ser comórbidas, potencializadas, ou também secundárias aos sintomas depressivos (Rinaldi, Gindri, Argimon, \& Fonseca, 2011).

Quando se refere a sintomas depressivos, a prevalência aumenta, tendo em vista que as escalas utilizadas para mensurar esses sintomas não preenchem os critérios suficientes para diagnosticar depressão de acordo com o DSM-IV e o CID-10 (Irigaray \& Schneider, 2007), variando, assim, de 6,4\% a 59,3\% (Frank \& Rodrigues, 2006). A prevalência de sintomatologia depressiva encontrada em 56 idosos da comunidade por Hamdan e Corrêa (2009) foi de 37,5\% e por Castro-Costa, LimaCosta, Carvalhais, Firmo, e Uchoa (2008) foi de 38,9\% entre 1510 idosos residentes na cidade de Bambuí (MG). Nos estudos de Bandeira (2008), com 85 idosos residentes da comunidade do município de Fortaleza (CE), e de Batistoni, Cupertino, e Neri (2009), com 903 idosos residentes na comunidade, foram encontradas frequência desses sintomas de $37,6 \%$ e $33,8 \%$, respectivamente. Alvarenga (2008) realizou um estudo com 503 idosos atendidos pela Estratégia de Saúde da Família de Dourados e constatou que $34,4 \%$ apresentaram sintomas depressivos. Além disso, por meio do modelo de regressão logística, verificou uma associação forte entre a presença de sintomatologia depressiva e as seguintes variáveis: inatividade econômica, ausência de atividade social, déficit cognitivo, avaliação ruim da saúde e baixa renda. O estudo de Ferreira e Tavares (2013) foi realizado com 850 idosos da zona rural e encontrou a prevalência de $22 \%$ de sintomatologia depressiva.

Um estudo realizado recentemente na cidade de Porto Alegre, com 621 idosos atendidos pela Estratégia de Saúde da Família, verificou que a prevalência de sintomas depressivos nessa amostra foi de 30,6\% (Nogueira, Rubin, Giacobbo, Gomes, \& Cataldo, 2014).
Steunenberg, Beekman, Deeg, e Kerkhof (2006) acompanharam 1511 idosos por seis anos e, destes, 17\% desenvolveram sintomas depressivos relevantes ao longo desse tempo. Essa variação de prevalência ocorre devido à variabilidade de instrumentos validados que são utilizados para rastrear e detectar os sintomas de depressão na velhice e aos diferentes pontos de corte estabelecidos para detectar esses sintomas (Araújo, 2012). Essa variação de prevalência pode ser atribuída ao contexto onde os estudos foram realizados e a diferentes instrumentos utilizados pelas pesquisas.

Fernandes et al (2012) apontam que os prejuízos cognitivos podem influenciar na visão da pessoa sobre si mesma, sobre o futuro e sobre o mundo. Portanto, ressalta-se a importância da avaliação dos sintomas depressivos concomitante à avaliação neuropsicológica global do idoso (Paula, Ribeiro, D'Elboux, \& Guariento, 2013), tendo em vista a possibilidade de eles estarem associados a um pior desempenho cognitivo (Silva et al., 2014). Uma pesquisa com 6376 mulheres idosas cognitivamente preservadas concluiu que a presença de sintomas depressivos se associa a um maior risco para o desenvolvimento de demência ou déficits cognitivos moderados (Goveas et al., 2012). Um outro estudo acompanhou 451 pessoas sem déficits cognitivos durante cinco anos e apontou que a presença de sintomatologia depressiva, independente de sua gravidade, associada ao prejuízo das funções executivas leva a um maior risco de desenvolvimento de demência, em especial da doença de Alzheimer (Vilalta-Franch et al., 2013). Royall, Palmer, Chiodo, e Polk (2012), em seu estudo, apontaram uma associação entre declínio das funções executivas e presença de sintomas depressivos. Diante disso, entende-se que é de grande relevância a avaliação das funções executivas em idosos com sintomas depressivos.

Grande parte dos estudos com pacientes deprimidos são voltados para a avaliação da memória em detrimento das demais funções cognitivas, tais como as funções executivas (Ávila \& Botitino, 2006). Entretanto, já nos estágios iniciais de um quadro depressivo, os pacientes podem apresentar deficiências executivas importantes que podem ser detectadas por testes que avaliam as funções executivas, como o Teste Wisconsin de Classificação de Cartas (WCST) (Lonen, Taiminen, \& Karlsson, 2000). Esse é um instrumento que possui reconhecimento internacional como sendo padrão ouro para a avaliação das funções executivas (Reppold, Pedrom, \& Trentini, 2010; Silva-Filho, Pasian, \& Barboza, 2013). A versão reduzida desse teste é denominada Teste Wisconsin de Classificação de Cartas versão 64 cartas (WCST-64) e tem se mostrado popular entre os pesquisadores que investigam o funcionamento executivo. Diante do exposto, o objetivo do presente estudo foi comparar o desempenho de idosos com e sem sintomas depressivos no WCST-64. Além disso, buscou-se verificar se existe uma associação entre os escores do WCST-64 e a quantidade de sintomas 
depressivos e analisar se diferentes grupos conforme a intensidade de sintomas depressivos apresentam diferenças no desempenho do WCST-64.

\section{Método}

\section{Delineamento}

Trata-se de um estudo transversal quantitativo.

\section{Participantes}

O estudo foi realizado com 371 idosos, de idade igual ou superior a 60 anos, de Porto Alegre e região metropolitana, Rio Grande do Sul, Brasil. Foram excluídos 61 idosos que não terminaram a aplicação dos instrumentos; 33 que obtiveram escores no Mini Exame do Estado Mental (MEEM, adaptado por Chaves \& Izquierdo, 1992) abaixo dos seguintes pontos de corte: 22 para cinco anos de escolaridade, 23 para escolaridade entre seis e 11 anos, e 24 para os indivíduos com 12 anos ou mais de estudo (Kochhann, Varela, Lisboa, \& Chaves, 2010); e um que apresentou problemas sensórios primários (auditivo e visual) não corrigidos durante o momento com da avaliação. Dos 276 idosos restantes, 53 apresentaram sintomas depressivos e foi possível fazer o pareamento de acordo com a idade, escolaridade e escore do MEEM, com uma variação de \pm 2 , de 51 desses idosos. Para realizar o pareamento, foi considerada a proporção de um idoso com sintomas de depressão para cada dois idosos sem tais sintomas.

Assim, a amostra foi composta por 153 idosos, sendo 51 com sintomas depressivos e 102 sem tais sintomas. Eles foram divididos em dois grupos: grupo caso (grupo 1) e grupo controle (grupo 2). O critério de inclusão para o grupo 1 foi a presença de sintomas depressivos, identificados por uma pontuação igual ou maior do que seis pontos na Escala de Depressão Geriátrica (GDS-15), versão composta por quinze itens (Yesavage et al., 1983). O grupo 2 teve como critério de inclusão a ausência de sintomas depressivos, que corresponde a um escore menor do que seis pontos na GDS-15.

\section{Instrumentos}

1. Ficha de dados sociodemográficos para caracterização da amostra que incluiu as variáveis sexo, idade, escolaridade, estado civil, coabitação, percepção de saúde e uso de medicação.

2. Mini Exame do Estado Mental (MEEM) para verificação do funcionamento cognitivo global. O MEEM, que é um instrumento de rastreio cognitivo e, nesse estudo, foi utilizado a fim de excluir os idosos com escores sugestivos de demência (Chaves \& Izquierdo, 1992).

3. Teste Wisconsin de Classificação de Cartas versão reduzida de 64 cartas (WCST-64) para mensurar as funções executivas, por ser um teste que avalia a flexibilidade cognitiva, o raciocínio abstrato, a inibição e a capacidade para gerar estratégias de solução de problemas (Trentini, Argimon, Oliveira, \& Werlang, 2010).
4. Escala de Depressão Geriátrica reduzida (GDS15), em português, composta por 15 questões para identificação e quantificação de sintomas depressivos nos idosos, que possui boa consistência interna com coeficiente alpha de Cronbach de 0,81 (Paradela, Lourenço, \& Veras, 2005; Yesavage et al., 1983). Além disso, é possível classificar a intensidade dos sintomas depressivos em leve (até 6 pontos), moderado (entre 7 e 10 pontos) e grave (a partir de 11 pontos). Este estudo adotou como ponto de corte o escore 5: indivíduos que obtiveram valores entre zero e 5 foram categorizados como normais (sem sintomas depressivos), enquanto que aqueles com escores maiores do que 5 foram classificados como possuindo sintomas depressivos (Paradela et al., 2005).

\section{Procedimentos}

Primeiramente, este estudo foi aprovado pelo Comitê de Ética em Pesquisa da Pontifícia Universidade Católica do Rio Grande do Sul (CAAE n ${ }^{\circ}$ 14769713.1.0000.5336). Os participantes foram recrutados por anúncios em jornais e indicação, por meio da técnica de amostragem não aleatória de conveniência. Antes do início das avaliações, os idosos preencheram e assinaram o Termo de Consentimento Livre e Esclarecido. Os instrumentos foram aplicados individualmente, em uma sessão de avaliação, com duração média de 90 minutos, em local apropriado.

\section{Análise de Dados}

A descrição dos dados foi realizada por meio de frequências absolutas (n) e relativas (\%) para variáveis qualitativas e, por média e desvio padrão, para variáveis quantitativas. Para todas as análises foram utilizados o software SPSS versão 20.0, considerando um nível de significância de 5\%.

Identificada a presença de distribuição normal das variáveis por meio do teste Kolmogorov-Smirnov, para análise dos dados, utilizou-se testes paramétricos. Foi realizado um teste $t$ de Student para amostras independentes para comparação entre os dois grupos (com e sem sintomas depressivos) e as variáveis: idade, anos de estudo, escore bruto do MEEM e as categorias do WCST-64. O tamanho de efeito foi calculado por meio do $d$ de Cohen. O Teste Qui-quadrado foi realizado para comparação das variáveis qualitativas entre os grupos. A associação entre os escores do WCST-64 e GDS15 foi realizada por meio da correlação de Pearson. Para analisar diferenças entre os grupos conforme a intensidade de sintomas depressivos (leve, moderado e grave) no desempenho do WCST-64, foi realizado o teste One Way Anova com post hoc Scheffe.

\section{Resultados}

De acordo com a Tabela 1, é possível observar os dados sociodemográficos da amostra total e a 
comparação entre os idosos com e sem sintomatologia depressiva e verifica-se que os grupos possuem características semelhantes. Conforme se pode observar, os dois grupos diferiram-se de maneira estatisticamente significativa somente em relação à percepção subjetiva de saúde, sendo que a maior parte dos indivíduos sem sintomatologia depressiva teve uma percepção de sua saúde como sendo boa $(72,5 \%)$ e os com sintomatologia como sendo regular $(51 \%)$.
Destaca-se que a maior parte da amostra pareada é formada por mulheres $(83,7 \%)$. O estado civil mais frequente foi casado ou com companheiro, tanto no grupo com sintomas depressivos (39,2\%), quanto no grupo sem esses sintomas (49\%), e a maioria faz uso de medicação, com uma frequência de $92,2 \%$ e $82,4 \%$, respectivamente. $\mathrm{Na}$ Tabela 1 , ainda pode se observar que a amostra se mostrou semelhante em relação à idade, anos de estudo e capacidade cognitiva geral.

Tabela 1

Sintomas Depressivos e Informações Sociodemográficas

\begin{tabular}{|c|c|c|c|c|}
\hline \multirow[b]{2}{*}{ Variáveis sociodemográficas } & \multicolumn{4}{|c|}{ Sintomas depressivos } \\
\hline & $\begin{array}{c}\text { Total da } \\
\text { amostra } \\
\text { pareada } \\
(n=153)\end{array}$ & $\begin{array}{c}\text { Grupo 1: } \\
\text { Presença } \\
(n=51)\end{array}$ & $\begin{array}{l}\text { Grupo 2: } \\
\text { Ausência } \\
(n=102)\end{array}$ & $p$ \\
\hline \multicolumn{5}{|l|}{ Sexo } \\
\hline Feminino & $128(83,7 \%)$ & $39(76,5 \%)$ & $89(87,3 \%)$ & \multirow{2}{*}{$0,089^{* *}$} \\
\hline Masculino & $25(16,3 \%)$ & $12(23,5 \%)$ & $13(12,7 \%)$ & \\
\hline \multicolumn{5}{|l|}{ Estado Civil } \\
\hline Casado(a) ou com companheiro(a) & $70(45,8 \%)$ & $20(39,2 \%)$ & $50(49 \%)$ & \multirow{4}{*}{$0,259^{* *}$} \\
\hline Viúvo(a) & $47(30,7 \%)$ & $14(27,4 \%)$ & $33(32,4 \%)$ & \\
\hline Separado(a) & $24(15,7 \%)$ & $11(21,6 \%)$ & $13(12,7 \%)$ & \\
\hline Solteiro(a) & $12(7,8 \%)$ & $6(11,8 \%)$ & $6(5,9 \%)$ & \\
\hline \multicolumn{5}{|l|}{ Mora com alguém } \\
\hline Sozinho(a) & $47(30,8 \%)$ & $16(31,4 \%)$ & $31(30,4 \%)$ & \multirow{5}{*}{$0,302^{* *}$} \\
\hline Com marido/esposa ou companheiro(a) & $38(24,8 \%)$ & $12(23,5 \%)$ & $26(25,5 \%)$ & \\
\hline Com marido/esposa ou companheiro(a) e com filhos(as) ou netos(as) & $36(23,5 \%)$ & $10(19,6 \%)$ & $26(25,5 \%)$ & \\
\hline Com filhos(as) ou netos(as) & $23(15,0 \%)$ & $7(13,7 \%)$ & $16(15,7 \%)$ & \\
\hline Outros & $9(5,9 \%)$ & $6(11,8 \%)$ & $3(2,9 \%)$ & \\
\hline \multicolumn{5}{|l|}{ Percepção da saúde } \\
\hline Boa & $96(62,7 \%)$ & $22(43,1 \%)$ & $74(72,5 \%)$ & \multirow{3}{*}{$<0,001^{* *}$} \\
\hline Regular & $54(35,3 \%)$ & $26(51,0 \%)$ & $28(27,5 \%)$ & \\
\hline Ruim & $3(2,0 \%)$ & $3(5,9 \%)$ & $0(0 \%)$ & \\
\hline \multicolumn{5}{|l|}{ Uso de medicação } \\
\hline $\operatorname{Sim}$ & $131(85,6 \%)$ & $47(92,2 \%)$ & $84(82,4 \%)$ & \multirow[t]{2}{*}{$0,103^{* *}$} \\
\hline Não & $22(14,4 \%)$ & $4(7,8 \%)$ & $18(17,6 \%)$ & \\
\hline Escore MEEM $(M \pm D P)$ & $27,64 \pm 1,91$ & $27,43 \pm 2,11$ & $27,75 \pm 1,8$ & $0,339^{*}$ \\
\hline Anos de estudo $(M \pm D P)$ & $9,78 \pm 4,32$ & $9,57 \pm 4,36$ & $9,88 \pm 4,32$ & $0,674^{*}$ \\
\hline Idade $(M \pm D P)$ & $68,63 \pm 5,57$ & $68,59 \pm 5,61$ & $68,66 \pm 5,58$ & $0,943^{*}$ \\
\hline
\end{tabular}

Nota. ${ }^{*}$ Teste t de Student. ${ }^{* *}$ Teste Qui-quadrado

De acordo com a Tabela 2, pode-se observar, por meio do teste de correlação de Pearson, uma correlação significativa positiva e fraca entre os sintomas depressivos e o total de erros, respostas e erros perseverativos no WCST-64. Esses resultados apontam que, na medida em que os sintomas de depressão se intensificam, aumentam o total de erros, as respostas e os erros perseverativos no WCST-64.

Pode-se observar, também, uma correlação significativa negativa e fraca entre os sintomas depressivos e o índice aprendendo a aprender, o número de respostas de nível conceitual e o de categorias completadas. 
Dessa maneira, na medida em que os sintomas depressivos aumentam, diminuem o índice aprendendo a aprender, o número de respostas de nível conceitual e o de categorias completadas, sugerindo que idosos com mais sintomas depressivos completam menos categorias no WCST-64 e apresentam menos respostas de nível conceitual e índices mais baixos de aprendendo a aprender.

Tabela 2

Correlação entre o Escore do GDS-15 e as Categorias do WCST-64

\begin{tabular}{cccccccccccc}
\hline & & TE & RP & EP & ENP & RNC & CC & TPC & RUP & AA \\
\hline & $R$ & $0,199^{*}$ & $0,263^{* *}$ & $0,278^{* *}$ & $-0,067$ & $-0,181^{*}$ & $-0,178^{*}$ & 0,155 & $-0,031$ & $-0,239^{*}$ \\
Escore GDS-15 & $p$ & 0,014 & 0,001 & 0,001 & 0,407 & 0,026 & 0,028 & 0,056 & 0,705 & 0,034 \\
& $n$ & 153 & 153 & 153 & 153 & 153 & 153 & 153 & 152 & 79 \\
\hline
\end{tabular}

Nota. Correlação de Pearson; ${ }^{*} p<0,05$ e ${ }^{* *} p<0,001$. TE=total de erros; RP=respostas perseverativas; EP=erros perseverativos; $\mathrm{ENP}=$ =rros não perseverativos; $\mathrm{RNC}=$ respostas de nível conceitual; $\mathrm{CC}=$ categorias completadas; TPC=tentativas para completar a primeira categoria; $\mathrm{RUP}=$ rupturas; $\mathrm{AA}=$ aprendendo a aprender

De acordo com a Tabela 3, os grupos diferenciaram-se de maneira estatisticamente significativa nos seguintes escores do WCST-64, mas com tamanho de efeito pequeno, em: quantidade de erros, de respostas perseverativas, de erros perseverativos e de categoriais completas, sendo que os idosos com sintomas depressivos obtiveram pior desempenho, comparados ao grupo controle. O número médio de erros cometidos pelos indivíduos com sintomas depressivos foi de 31,33 ( $D P \pm 11,21)$, correspondendo a quase metade do total das 64 cartas; já os sem sintomas tiveram uma média de 27,69 $(D P \pm 10,12)$ erros, sendo a maioria desses erros perseverativos em ambos os grupos, com uma média de 18,57 $(D P \pm 10,49)$ e 15,06 $(D P \pm 8,85)$, respectivamente. Esses erros perseverativos podem refletir inflexibilidade e resistência à mudança durante a execução da tarefa. As pessoas com tal sintomatologia completaram em média $1,49(D P \pm 1,17)$ categorias, e as sem esses sintomas completaram, em média, $1,94(D P \pm 1,24)$. Diante de tal resultado, pode-se inferir que os idosos que apresentaram sintomas depressivos, em comparação ao grupo controle, fizeram mais erros e, com isso, completaram menos categorias.

Tabela 3

Idosos com e sem Sintomas Depressivos: Comparação o Desempenho no WCST-64

\begin{tabular}{|c|c|c|c|c|c|c|c|}
\hline \multirow[t]{2}{*}{$\begin{array}{l}\text { Indicadores } \\
\text { WCST-64 }\end{array}$} & \multicolumn{2}{|c|}{$\begin{array}{c}\text { Grupo 1: } \\
\text { com sintomas } \\
\text { depressivos } \\
(n=51)\end{array}$} & \multicolumn{2}{|c|}{$\begin{array}{c}\text { Grupo 2: } \\
\text { sem sintomas } \\
\text { depressivos } \\
(n=102)\end{array}$} & \multirow[t]{2}{*}{ F } & \multirow[t]{2}{*}{$p$} & \multirow[t]{2}{*}{$\begin{array}{l}d \text { de } \\
\text { Cohen }\end{array}$} \\
\hline & $M$ & $\mathrm{DP}$ & M & $D P$ & & & \\
\hline Total de erros & 31,33 & 11,21 & 27,69 & 10,12 & 0,262 & $0,045^{*}$ & 0,341 \\
\hline Respostas perseverativas & 22,27 & 13,98 & 17,90 & 12,17 & 1,922 & $0,048^{*}$ & 0,333 \\
\hline Erros perseverativos & 18,57 & 10,49 & 15,06 & 8,85 & 2,581 & $0,032^{*}$ & 0,362 \\
\hline Erros não perseverativos & 12,76 & 8,72 & 12,64 & 7,79 & 0,125 & 0,927 & 0,014 \\
\hline Número de respostas de nível conceitual & 24,31 & 13,88 & 28,55 & 13,61 & 0,070 & 0,073 & $-0,308$ \\
\hline Categorias completas & 1,49 & 1,17 & 1,94 & 1,24 & 0,011 & $0,033^{*}$ & $-0,373$ \\
\hline Tentativas para formar a primeira categoria & 28,47 & 22,57 & 22,75 & 18,99 & 5,601 & 0,101 & 0,274 \\
\hline Rupturas & 0,74 & 1,74 & 0,50 & 1,35 & 0,417 & 0,581 & 0,154 \\
\hline Aprendendo a aprender & $-19,90$ & 16,23 & $-15,57$ & 15,24 & 0,425 & 0,269 & $-0,275$ \\
\hline
\end{tabular}

Nota. Teste t de Student; ${ }^{*} p<0,05$

De acordo com a Tabela 4, é possível observar que não houve diferença estatisticamente significativa entre nenhum escore no WCST-64, quando comparados os grupos com sintomas depressivos de intensidade leve a moderada com os de intensidade grave. Assim, é possível observar que a intensidade de sintomas depressivos não afetou de maneira significativa o desempenho dos idosos no WCST-64. 
Tabela 4

Desempenho no WCST-64 de Acordo com a Intensidade de Sintomas Depressivos

\begin{tabular}{|c|c|c|c|c|c|c|c|c|}
\hline \multirow{2}{*}{$\begin{array}{l}\text { Indicadores } \\
\text { WCST-64 }\end{array}$} & \multicolumn{2}{|c|}{$\begin{array}{l}\text { Leve } \\
(n=26)\end{array}$} & \multicolumn{2}{|c|}{$\begin{array}{c}\text { Moderada } \\
\quad(n=17)\end{array}$} & \multicolumn{2}{|c|}{$\begin{array}{l}\text { Grave } \\
(n=8)\end{array}$} & \multirow[t]{2}{*}{$F$} & \multirow{2}{*}{$p$} \\
\hline & $M$ & $\mathrm{DP}$ & $M$ & $\mathrm{DP}$ & $M$ & $\mathrm{DP}$ & & \\
\hline Total de erros & 30,92 & 11,19 & 30,65 & 11,96 & 34,13 & 10,66 & 0,289 & 0,751 \\
\hline Respostas perseverativas & 21,73 & 14,11 & 19,65 & 11,47 & 29,63 & 17,47 & 1,451 & 0,244 \\
\hline Erros perseverativos & 17,88 & 10,49 & 17,00 & 9,10 & 24,13 & 12,70 & 1,388 & 0,259 \\
\hline Erros não perseverativos & 13,04 & 10,37 & 13,65 & 7,33 & 10,00 & 5,07 & 0,492 & 0,615 \\
\hline Número de respostas de nível conceitual & 25,08 & 13,20 & 25,06 & 15,41 & 20,25 & 13,73 & 0,397 & 0,675 \\
\hline Categorias completas & 1,50 & 1,14 & 1,65 & 1,32 & 1,13 & 0,99 & 0,531 & 0,591 \\
\hline Tentativas para formar a primeira categoria & 26,38 & 20,26 & 29,29 & 24,76 & 33,50 & 26,89 & 0,312 & 0,733 \\
\hline Rupturas & 0,96 & 2,3 & 0,59 & 0,94 & 0,38 & 0,74 & 0,432 & 0,651 \\
\hline Aprendendo a aprender & $-13,29$ & 15,01 & $-23,22$ & 16,92 & $-28,67$ & 14,45 & 1,455 & 0,258 \\
\hline
\end{tabular}

Nota. One Way Anova

\section{Discussão}

O presente estudo teve como principal objetivo analisar a associação entre sintomas depressivos na velhice e funcionamento executivo, por meio do teste WCST-64. De maneira geral, os resultados mostraram que os idosos que apresentam sintomatologia depressiva tiveram pior desempenho no WCST-64, quando comparados com os sem essa condição, confirmando a hipótese inicial desse estudo, embora o tamanho de efeito dessa diferença tenha sido pequeno.

Os resultados do presente estudo são reforçados pelos do estudo de Lonen et al. (2000), que observaram que 97 pacientes pareados por idade e escolaridade que apresentavam um quadro depressivo mais grave demonstraram importantes prejuízos no desempenho do Teste Wisconsin de Classificação de Cartas (WCST). Feil, Razani, Boone, e Lesser (2003) também corroboram com esse achado, ao relatarem que, em seu estudo, os idosos foram avaliados por meio do WCST e o Teste de Stroop, e os que tinham depressão apresentaram um declínio nas funções executivas. Wagner (2003) corrobora com a ideia de que os sintomas depressivos influenciam no desempenho das funções executivas e ainda salienta que, ao serem retiradas as influências dos sintomas de depressão nas funções executivas, as diferenças no desempenho no WCST entre os grupos sem e com declínio cognitivo leve diminuem. Tal resultado pode ser explicado pelo fato de a depressão ser considerada como um fator de risco para o declínio cognitivo (Steibel \& Almeida, 2010). Outros estudos confirmam essa teoria com resultados semelhantes, apontando correlações entre a presença de sintomas depressivos e pior desempenho cognitivo (Alvarenga, 2008; Ávila \& Bottino, 2006; Rinaldi et al., 2011). Esse resultado também é corroborado por Ávila e Bottino (2006), que referem que a depressão reduz o desempenho de idosos nos testes de memória e, principalmente, nos de funções executivas.
Outras pesquisas também corroboram com o achado do presente estudo de que a presença de sintomas depressivos ocasiona um prejuízo no desempenho das funções executivas (Elderkin-Thompson et al., 2003; Esteves, 2012; Ferreira, 2012; Rapp et al., 2005).

Os achados mostraram que o grupo com sintomas depressivos obteve média mais baixa em todos os escores do WCST-64 quando comparado com o grupo sem sintomas depressivos, indicando um pior desempenho no teste. Entretanto, os dois grupos diferenciaram-se de maneira estatisticamente significativa somente nos seguintes escores: quantidade de erros, respostas perseverativas, erros perseverativos e categoriais completadas. Diante desses resultados, salienta-se que a média de erros cometidos pelos idosos com sintomas de depressão aponta que menos da metade das cartas foram classificadas corretamente, o que indica que houve uma maior dificuldade por parte dos idosos com sintomas depressivos para realização do teste. Maiores índices de erros indicaram que os idosos com sintomas depressivos sinalizaram menor flexibilidade na busca de novos modos de categorização das cartas, revelando pouco aproveitamento do feedback do examinador. Corroborando com esse resultado, um estudo realizado por Nascimento (2007) identificou um maior número de erros no WCST em idosos com depressão mais grave. Em um estudo também realizado na cidade de Porto Alegre, foram avaliados idosos por meio do WCST, e o grupo com sintomas depressivos apresentou um maior número de erros, quando comparado ao grupo sem tal sintomatologia (Lopes, Nascimento, Esteves, Terroso, \& Argimon, 2013).

O presente estudo também encontrou diferença significativa entre os grupos em relação ao total de categorias completadas, com pior desempenho pelo grupo com sintomas depressivos e maior número de tentativas para completar a primeira categoria. Esses resultados são corroborados por um estudo que encontrou um número significativamente reduzido de categorias completadas 
em adultos com depressão, quando comparado com adultos sem esse transtorno, além de maior número de ensaios para completar a primeira categoria no WCST (Totić-Poznanović, Marinković, Pavlović, Tomić, \& Paunović, 2006). Uma menor quantidade de categorias completadas indicou que os idosos com sintomas de depressão apresentaram pior capacidade de resolução de problemas, apresentando dificuldades para descobrir os critérios corretos de classificação das cartas e executarem com sucesso a tarefa proposta por esse teste.

A diferença significativa encontrada entre os grupos em relação aos erros perseverativos e respostas perseverativas pode refletir resistência à mudança e inflexibilidade na tarefa realizada (Lopes et al., 2013) por parte do grupo com sintomas depressivos, conforme os achados do presente estudo, o que pode ter ocorrido devido às próprias características dos sintomas depressivos (que reduzem a motivação e os recursos cognitivos), indicando a não experimentação de novas alternativas para solução do problema. Ou seja, disso entende-se que houve pouco aproveitamento do feedback do avaliador, havendo menor flexibilidade mental, tendo em vista reduzida variação das estratégias utilizadas para a categorização das cartas.

Em relação aos dados obtidos, dentre os idosos que apresentaram sintomatologia depressiva, a maioria era do sexo feminino. Esse dado é corroborado por outras pesquisas com idosos que também encontraram maior prevalência de mulheres com sintomas depressivos (Alvarenga, 2008; Bandeira, 2008; Batistoni et al., 2009; Castro-Costa et al., 2008; Nardi \& Andrade, 2005; Paula et al., 2013). Ferreira e Tavares (2013) ainda acrescentam que, de acordo com seus achados, o sexo feminino apresentou 53\% mais chances de possuir esses sintomas. Uma das possíveis explicações para o maior acometimento das mulheres pode estar relacionado a alterações hormonais, tais como diminuição dos hormônios sexuais produzidos pelos ovários, que ocorrem como consequência da transição do período fértil para o não reprodutivo (Polisseni, Polisseni, Fernandes, Moraes, \& Guerra, 2009).

Os resultados também mostraram que a intensidade dos sintomas depressivos não afetou o desempenho dos idosos no WCST-64. Disso, infere-se que a gravidade dos sintomas depressivos parece não alterar o desempenho executivo de idosos, mas sim a presença de sintomatologia. Assim, verificou-se que uma pontuação acima de cinco pontos na GDS-15 já interfere no desempenho executivo dos idosos, independentemente da intensidade dos sintomas depressivos, ou seja, leve, moderada ou grave. Rozhental, Laks, e Engelhardt (2004) apontam que a presença de sintomatologia depressiva é também manifestada por meio da sonolência e da fadiga, que são capazes de afetar o nível de atenção e, consequentemente, prejudicar o desempenho em tarefas cognitivas.

Os resultados do presente estudo demonstram sua importância na medida em que apontam que a presença de sintomas depressivos ocasiona um prejuízo no desempenho das funções executivas, medido por meio do desempenho de idosos da população brasileira no WCST64, comparação ainda não existente na literatura. Por ser uma versão reduzida, esse teste torna-se mais eficaz à prática clínica e à pesquisa por despender menos tempo de aplicação do que a versão ampliada, além de gerar menos estresse e frustração aos indivíduos por ele avaliados.

Constitui uma limitação o fato de o presente estudo ter sido conduzido com uma amostra que apresentava características relativamente homogêneas e com um nível de escolaridade elevado. Sugere-se que novos estudos sejam realizados com amostras mais diversificadas e que também visem aprofundar os dados encontrados a respeito de que somente a presença de sintomas depressivos já pode causar prejuízos no desempenho de idosos no WCST-64, e não a intensidade de sintomas depressivos. Assim, salienta-se a necessidade de aumentar a amostra estudada, realizando estudos com grupos comparativos de participantes que apresentem sintomas leves, moderados e graves de depressão.

\section{Referências}

Alvarenga, M. R. M. (2008). Avaliação da capacidade funcional, do estado de saúde e da rede de suporte social do idoso atendido na Atenção Básica (Tese de doutorado, Universidade de São Paulo). Recuperado de http://www.teses.usp.br/teses/disponiveis/7/7136/tde-07052009-083059/ pt-br.php

Araújo, J. D. (2012). Polarização epidemiológica no Brasil. Epidemiologia e Serviços Saúde, 21(4), 533-538. doi: 10.5123/S167949742012000400002

Ávila, R., \& Bottino, C. M. C. (2006). Atualização sobre alterações cognitivas em idosos com síndrome depressiva. Revista Brasileira de Psiquiatria, 28(4), 316-320. doi: 10.1590/S1516-44462006005000010

Bandeira, C. B. (2008). Perfil dos idosos com depressão em comunidade do município de Fortaleza. Revista Brasileira de Medicina da Família e Comunidade, 4(15), 189-120. doi: 10.5712/rbmfc4(15)171

Batistoni, S. S. T., Cupertino, A. P. F. B., \& Neri, A. L. (2009). Sintomas depressivos e variáveis psicossociais em idosos residentes na comunidade. Geriatria \& Gerontologia, 3(2), 78-84.

Castro-Costa, E., Lima-Costa, M. F., Carvalhais, S., Firmo, J., \& Uchoa, E. (2008). Factors associated with depressive symptoms measured by the 12-item general health questionnaire in community-dwelling older adults (The Bambuí Health Aging Study). Revista Brasileira de Psiquiatria, 30(2), 104-109. doi: 10.1590/\$1516-44462008005000007 
Chaves, M. L., \& Izquierdo, I. (1992). Differential diagnosis between dementia and depression: A study of efficiency increment. Acta Neurologica Scandinavica, 85(6), 378-382.

Elderkin-Thompson, V., Kumar, A., Bilker, W. B., Dunkin, J. J., Mintz, J., Moberg P. J. ... Gur, R. E. (2003). Neuropsychological deficits among patients with late-onset minor and major depression. Archives of Clinical Neuropsychology, 18(5), 529-549.

Esteves, C. S. (2012). Desempenho cognitivo de idosos atendidos pelo programa estratégia da saúde da família (ESF) (Dissertação de mestrado, Pontifícia Universidade Católica do Rio Grande do Sul, Porto Alegre). Recuperado de http://hdl.handle.net/10923/4884

Feil, D., Razani, J., Boone, K., \& Lesser, I. (2003). Apathy and cognitive performance in older adults with depression. International Journal of Geriatric Psychiatry, 18(6), 479-485. doi: 10.1002/gps.869

Fernandes, C. S., Falcone, E. M. O., \& Sardinha, A. (2012). Deficiências em habilidades sociais na depressão: estudo comparativo. Psicologia: Teoria e Prática, 14(1), 183-196.

Ferreira, L. S. (2012). O impacto das funções executivas no funcionamento cognitivo do idoso sob resposta social (Dissertação de mestrado). Recuperado de http://repositorio.ismt.pt/xmlui/handle/123456789/444

Ferreira, P. C. S., \& Tavares, D. M. S. (2013). Prevalência e fatores associados ao indicativo de depressão entre idosos residentes na zona rural. Revista da Escola de Enfermagem da USP, 47(2), 401-407. doi: 10.1590/S0080-62342013000200018

Frank, M. H., \& Rodrigues, N. L. (2006). Depressão, ansiedade, outros distúrbios afetivos e suicídio. Em E. V. Freitas, L. Py, A. L. Neri, F. A. X. Cançado, J. Doll, \& M. L. Gorzoni (Eds.), Tratado de geriatria e gerontologia (pp. 376-387). Rio de Janeiro: Guanabara.

Garcia, A., Passos, A., Campo, A. T., Pinheiro, E., Barroso, F., Coutinho, G. ... Sholl-Franco, A. (2006). A depressão e o processo de envelhecimento. Ciência \& Cognição, 7, 111-121.

Goveas, J. S., Hogan, P. E., Kotchen, J. M., Smoller, J. W., Denburg, N. L., Manson, J. E. ... Wassertheil-Smoller, S. (2012). Depressive symptoms, antidepressant use, and future cognitive health in postmenopausal women: The Women's Health Initiative Memory Study. International Psychogeriatrics/IPA, 24(8), 1252-1264. doi: 10.1017/S1041610211002778

Gunn, J. M., Ayton, D. R., Densley, K., Pallant, J. F., Chondros, P., Herrman, H. E., \& Dowrick, C. F. (2012). The association between chronic illness, multimorbidity and depressive symptoms in an Australian primary care cohort. Social Psychiatry and Psychiatric Epidemiology, 47(2), 175-184. doi: 10.1007/s00127-010-0330-z

Hamdan, A. C., \& Corrêa, P. H. (2009). Memória episódica e funções executivas em idosos com sintomas depressivos. Psico, 40(1), 73-80.

Irigaray, T. Q., \& Scheneider, R. H. (2007). Características de personalidade e depressão em idosas da Universidade para a Terceira Idade (UNITI/UFRGS). Revista de Psiquiatria do Rio Grande do Sul, 29(2), 169-175. doi: 10.1590/S0101-81082007000200008

Kochhann, R., Varela, J., Lisboa, C. S. M., \& Chaves, M. L. (2010). The Mini Mental State Examination Review of cutoff points adjusted for schooling in a large southern Brazilian sample. Dementia \& Neuropsychologia, 4(1), 35-41.

Lonen, T., Taiminen, T., \& Karlsson, H. (2000). Impaired wisconsin card sorting teste performance in first-episode severe depression. Nordic Journal of Psychiatry, 54(4), 275-280. doi: 10.1080/080394800448156

Lopes, R. M. F., Nascimento, R. F. L., Esteves, C. S., Terroso, L. B., \& Argimon, I. I. L. (2013). Funções executivas de idosos com depressão: um estudo comparativo. Cuadernos de Neuropsicología, 7(2), 72-86. doi: 10.7714/cnps/7.2.204

Nardi, E. F. R., \& Andrade, O. G. (2005). Estados depressivos entre idosos na comunidade - Jandaia do Sul, Paraná, Brasil. Arquivos de Ciência da Saúde da UNIPAR, 9(2), 109-116.

Nascimento, R. F. L. (2007). Depressão e o desempenho cognitivo em idosos através do teste Wisconsin Classificação de Cartas (Dissertação de mestrado). Recuperado de http://hdl.handle.net/10923/4871

Nogueira, E. L., Rubin, L. L., Giacobbo, S. S., Gomes, I., \& Cataldo, A. C. (2014). Rastreamento de sintomas depressivos em idosos na estratégia saúde da família, Porto Alegre. Revista de Saúde Pública, 48(3), 368-377. doi: 10.1590/S0034-8910.2014048004660

Paradela, E. M. P., Lourenço, R. A., \& Veras, R. P. (2005). Validação da escala de depressão geriátrica em um ambulatório geral. Revista de Saúde Pública, 39(6), 918-923. doi: 10.1590/S0034-89102005000600008

Paula, A. F. M., Ribeiro, L. H. M., D’Elboux, M. J., \& Guariento, M. E. (2013). Avaliação da capacidade funcional, cognição e sintomatologia depressiva em idosos atendidos em ambulatório de Geriatria. Revista da Sociedade Brasileira de Clínica Médica, 11(3), 212-218.

Polisseni, A. F., Polisseni, F., Fernandes, L. M., Moraes, M. A., \& Guerra, M. O. (2009). Depressão em mulheres climatéricas: fatores associados. Revista Brasileira de Ginecologia e Obstetrícia, 35(3), 183-189.

Rapp, M. A., Dahlman, K., Sano, M., Grossman, H. T., Haroutunian, V., \& Gorman J. M. (2005). Neuropsychological differences between late-onset and recurrent geriatric major depression. American Journal of Psychiatry, 162(4), 691-698.

Reppold, C. T., Pedrom, A. C., \& Trentini, C. M. (2010). Avaliação das funções executivas por meio do Teste Wisconsin de Classificação de Cartas - versão computadorizada. Em M. C. R. A. Joly \& C. T. Reppold (Eds.), Estudos de testes informatizados para avaliação psicológica (pp. 45-62). São Paulo. Capsi Livraria e Editora Ltda.

Rinaldi, J., Gindri, G., Argimon, I. I. L., \& Fonseca, R. P. (2011). Perfil neuropsicológico de idosas com sintomas depressivos. A terceira idade, 22(52), 33-51.

Royall, D. R., Palmer, R., Chiodo, L. K., \& Polk, M. J. (2012). Depressive symptoms predict longitudinal change in executive control but not memory. International Journal of Geriatric Psychiatry, 27(1), 89-96. doi: 10.1002/gps.2697

Rozenthal, M., Laks, J., \& Engelhardt, E. (2004). Aspectos neuropsicológicos da depressão. Revista de Psiquiatria do Rio Grande do Sul, 26(2), 204-212.

Santos, E. G., \& Siqueira, M. M. (2010). Prevalência dos transtornos mentais na população adulta brasileira: uma revisão sistemática de 1997 a 2009. Jornal Brasileiro de Psiquiatria, 59(3), 238-246.

Silva, L. S. V., Silva, T. B. L., Falcão, D. V. S., Batistoni, S. S. T., Lopes, A., Cachioni, M.,... Yassuda, M. S. (2014). Relações entre queixas de memória, sintomas depressivos e desempenho cognitivo em idosos residentes na comunidade. Revista de Psiquiatria Clínica, 41(3), 67-71. doi: 10.1590/0101-60830000000013

Silva-Filho, J. H., Pasian, S. R., \& Barboza, L. L. (2013). Potencial informativo e desafios técnicos do Teste Wisconsin de Classificação de Cartas. Revista da SPAGESP, 14(2), 102-113.

Steibel, N. M., \& Almeida, R. M. M. (2010). Estudo de caso-avaliação neuropsicológica: depressão x demência. Aletheia, 31, 111-120.

Steunenberg, B., Beekman A. T., Deeg D. J., \& Kerkhof A. J. (2006). Personality and the onset of depression in late life. Journal of Affective Disorders, 92(2-3), 243-251.

Totić-Poznanović, S., Marinković, D., Pavlović, D., Tomić, G., Paunović, \& V. R. (2006). Executive functions in young patients with unipolar depression. Safety Lit Journal Details, 134(7-8), 273-277. 
Trentini, C. M., Argimon, I. I. L., Oliveira, M. S., \& Werlang, B. G. (2010). Teste Wisconsin de Classificação de Cartas: versão para idosos - adaptação e padronização brasileira. São Paulo: Casa do Psicólogo.

Vilalta-Franch, J., Lopez-Pousa, S., Llinas-Regla, J., Calvo-Perxas, L., Merino-Aguado, J., \& Garre-Olmo, J. (2013). Depression subtypes and 5-year risk of dementia and Alzheimer disease in patients aged 70 years. International Journal of Geriatric Psychiatry, 28(4), 341-350. doi: $10.1002 / \mathrm{gps}$

Wagner, G. P. (2003). Disfunções executivas no envelhecimento cognitivo: Investigações com os instrumentos tarefa do jogo e Teste Wisconsin de Classificação de Cartas (Dissertação de mestrado). Recuperado de http://hdl.handle.net/10183/11247

Xavier, F. M. F. (2006). O transtorno cognitivo da depressão ou "pseudodemência depressiva" do idoso. Em M. A. M. P. Parente (Ed.), Cognição e Envelhecimento (pp. 203-224). Porto Alegre: Artmed.

Yesavage, J. A., Brink, T. L., Rose, T. L., Lum, O., Huang, V., Adey, M., \& Leirer, V. O. (1983). Development and validation of a geriatric depression screening scale: a preliminary report. Journal of Psychiatric Research, 17(1), 37-39.

\section{Sobre as autoras}

Cristiane Silva Esteves é psicóloga, mestre em Psicologia Clínica (PUCRS) e doutoranda em Gerontologia biomédica (PUCRS).

Camila Rosa de Oliveira é psicóloga, mestre em Psicologia Clínica (PUCRS) e doutoranda em Gerontologia Biomédica (PUCRS).

Tatiana Quarti Irigaray é psicóloga, especialista em Psicologia Clínica, doutora em Gerontologia Biomédica (PUCRS), professora titular da Pontifícia Universidade Católica do Rio Grande do Sul.

Irani Iracema de Lima Argimon é psicóloga, mestre em educação, doutora em Psicologia Clínica (PUCRS), professora titular da Pontifícia Universidade Católica do Rio Grande do Sul. 